\title{
Genome Wide Association Analysis of Acid Detergent Fiber Content of 206 Forage Sorghum (Sorghum Bicolor (L.) Moench) Accessions
}

\author{
Hao Niu \\ Shanxi Agricultural University \\ junai ping \\ Shanxi Agricultural University \\ Yubin Wang \\ Shanxi Agricultural University \\ Xin Lv \\ Shanxi Agricultural University \\ Jianqiang Chu \\ Shanxi Agricultural University
}

Shanxi Agricultural University https://orcid.org/0000-0002-9656-4497

Yuanhuai Han ( $\square$ nkyglsnh@126.com )

\section{Research Article}

Keywords: Forage sorghum, Quantitative trait locus, acid detergent fiber content, Genome-wide association analysis

Posted Date: June 3rd, 2021

DOI: https://doi.org/10.21203/rs.3.rs-551963/v1

License: (c) (i) This work is licensed under a Creative Commons Attribution 4.0 International License. Read Full License

Version of Record: A version of this preprint was published at Genetic Resources and Crop Evolution on February 25th, 2022. See the published version at https://doi.org/10.1007/s10722-022-01355-0. 


\section{Abstract}

Forage Sorghum (Sorghum bicolor (L.) Moench) is an annual gramineous forage, which is a high-quality C4 crop. It is widely planted with Forage Maize in the world, especially in the developed countries of animal husbandry. Many of its important physiological characteristics vary with germplasm and growth environment. Acid detergent fiber is a kind of carbohydrate insoluble in acid detergent in plant material or feed containing plant material, including pure cellulose and acid cellulose. Acid detergent fiber widely exists in the stem and leaf tissues of forage sorghum, which is the main substance affecting the digestibility of livestock. It has a very important impact on the milk yield and quality of cows, dairy sheeps and pigs. In order to further understand its genetic mechanism, we re-sequenced 206 Forage Sorghum Germplasms from different regions of the world and identified 14,570,430 SNPs and 1,967,033 indels. Based on SNP markers, we analyzed the population genetic structure and identified the gene loci related to acid detergent fiber content by genome-wide association analysis (GWAS). Genetic relationship between materials showed that Asian and American sorghum varieties, breeding lines and improved varieties were more diverse, while European varieties were relatively more closely related. These findings provide new clues and directions for further study. Eight QTLs harboring 91 genes were found to be associated with acid detergent fiber content. These genes were significantly enriched into 6 major genes involved in cell membrane material transport or enzymes, showing a regional distribution. This finding provides a basis for us to understand the origin and spread of haplotypes related to acid detergent fiber content, which would accelerate the study of genetic gain of acid detergent fiber content, and help breeders improve feed quality and stress tolerance of forage sorghum.

\section{Introduction}

Sorghum (Sorghum bicolor (L.) Moench) is one of the main food crops in the world, which can be divided into grain sweet, broom and forage sorghum. Different from the other three types, forage sorghum is suitable for planting in the poor soils not suitable for food crops, because of its strong growth ability, high yield, rich protein and easy digestion (National Research Council 1996; Vermerris 2011; Liu et al. 2018; Mundia et al. 2019). Forage sorghum is mainly used as fresh chop (Shoemaker and Bransby 2010), hay and silage for livestock, and is also widely used in the field of fishery breeding (Ping et al. 2018). In recent years, it has been exploited as a potential species for the production of sugar and lignocellulose biofuels (Mathur et al. 2017). Many scientists have made efforts to breed multi-purpose forage sorghum varieties or hybrids with good stem quality, high genetic stability, high sugar content, high dry matter yield and low hydrocyanic acid content by using traditional and biotechnological methods (Ping et al.2018).

The content of acid detergent fiber (ADF) is one of the main indexes to evaluate dietary fiber(Yu et al. 2017; Sara et al.2019; Miranda et al. 2020), the effect of acid detergent fiber concentration on nutrient digestibility and nitrogen balance were huge. In addition, the study on the effect of ADF on pig and cattle feeding showed that the digestibility of conventional nutrients and N-balance of pigs were significantly negatively correlated with ADF level (Yu et al.2017, Yu et al. 2018). Adding appropriate content of ADF can improve the production performance of pigs (Zhang et al. 2020; Luo et al. 2000; Yuan et al. 1999; Yu et al.2018). ADF content in barley (hordim commodiummailable L.) grains is a major index of digestibility, which has a negative impact on feed quality, especially for non-ruminant livestock and poultry (Han et al. 2003). At present, researchers mainly focus on the measurement method of ADF content and the feeding effect of different ADF content feed on livestock (He 2011; Font et al.2015) However, the molecular genetic mechanism of ADF content in forage sorghum has not been elucidated.

In recent years, the whole genome deep sequencing of sorghum population has been increasingly used for gene mapping analysis of target traits (Casa et al. 2008; NIDA et al. 2021; Boddu et al. 2001; Bouche et al. 2017; Mathur et al. 2017). For example, the whole genome single nucleotide diversity (Casa et al. 2006; Zheng et al. 2011; Bouche et al. 2012) and association analysis have been conducted by Morris et al. (2013) and Nida et al (2021). However, compared with other cereal crops such as corn (Ross et al.2017; Jiao et al. 2017; Ma et al. 2021), rice (Verma 2021; Huang et al. 2010), sorghum studies are scarce. Recently, two sweet sorghum restorer lines and one grain sorghum restorer line were re sequenced, and 1500 genes were identified to distinguish the two types of sorghum. Zheng et al. (2011) Morris et al. (2013) analyzed the diversity of a sorghum panel of 971 accessions genotyped by genotyping-by-sequencing (GBS) and several typical plant height and inflorescence structure loci and genes were mapped by GWAS. Genetic diversity of 48 Forage Sorghum Germplasm resources were analyzed by 109 SSRs (Ping et al. 2018). Zhang et al. (2018) analyzed the narrow sense genetic diversity of 21 introduced sorghum BMR male sterile lines based on SRAP. In order to further analyze the genetic diversity of forage sorghum, we re-sequenced 206 forage sorghum accessions from different geographical regions to explored the key genes related to acid detergent fiber content of Forage Sorghum by GWAS.

\section{Materials And Methods Plant materials}

We selected 206 forage sorghum, including 30 pairs of male sterile lines and 146 restorer lines. These materials have different genetic backgrounds and geographical regions, and are highly representative. 
The 206 forage sorghum were planted in Dongbai experimental station of Jinzhong Shanxi China, from 2016 to 2018 . We used a randomized block design with three replications. Plots were $5 \mathrm{~m}$ in length and $2.6 \mathrm{~m}$ in width 6rows per plot. At the end of August every year, 206 forage sorghum whole plants were harvested, each whole plant was chopped into $2-3 \mathrm{~cm}$ fragments according to the number and take $500 \mathrm{~g}$ for each variety in Parchment bags.. The samples were first dried at $105^{\circ} \mathrm{Cfor} 1$ hours, then lower the oven temperature to $65^{\circ} \mathrm{C}$ and continue to bake for 72 hours, at the end of all procedures, the dried samples were sent to Fine Tune Bio Technology Limited for quality traits analyzed. We tested 19 agronomic traits and 18 quality traits of 206 forage sorghum samples, including acid detergent fiber.

\section{Determination of ADF content}

NIRS technology was used to analyze the nutritional components of forage. Take $100 \mathrm{~g}$ of each sample to be tested, further crush it with a cyclone mill, pass through a $1 \mathrm{~mm}$ sieve, and conduct spectral scanning with FOSS 5000 near infrared analyzer (FOSS company, Denmark) (the working parameters are: wavelength range: $1100-2500 \mathrm{~nm}$, scanning times: 32 , spectral interval: $2 \mathrm{~nm}$ ), Based on the near infrared rapid detection model of Alfalfa from Cumberland Valley analytical services (CVAs), the dry matter (DM) and acid detergent fiber(ADF) in the samples were obtained.

\section{DNA isolation and genome sequencing}

DNA was extracted from forage sorghum by CTAB method (Murray and Thompson 1980) for genome sequencing on the lllumina HiSeq system (Personal Bio, Shanghai, China). Briefly, after the quality assessment, the genomic DNA was separated by ultrasonic fragmentation and electrophoresis, and then the DNA fragment of the desired length (400 bp) was purified by gel. The library with 400 inserts was constructed and sequenced by next generation sequencing (NGS) based on illumine Hiseq.

\section{Read alignment and variation calling}

After DNA extraction, purification, database construction and sequencing, the Raw data were obtained and saved in the format of paired end fastq, Clean reads were mapped to the reference genome BTx623 (v3.1) (McCormick et al. 2018)

(ftp://ftp.ncbi.nlm.nih.gov/genomes/genbank/plant/Sorghum_bicolor/latest_assembly_versions/GCA_000003195.3_Sorghum_bicolor_NCBIv3)

We used the software package of genome analysis TK v3.8 to detect SNP, In order to ensure the accuracy of SNP sites, SNP sites were filtered by Fisher test of strand bias (FS) $\leq 60$, Haplotype Score $\leq 13.0$, Mapping Quality (MQ) $\geq 40$, Quality Depth(QD) $\geq 2$, Read Pos Rank $\geq-8.0$, Mapping Quality Rank >-12.5.. Indels were filtrated with a Fisher Test of Strand Bias (FS) $\leq 200$, Quality Depth (QD) $\geq 2$, and Read Pos Rank $\geq$ -20 .

\section{Population genetics analysis and GWAS}

Based on SNPs among populations and linkage disequilibrium, markers or candidate genes closely related to target traits were identified by correlation analysis of molecular markers and phenotypes (analysis software EmMax (kanhm et al., 2010). 100kb before and after the point with - $\log 10$ ( $P$ value) $>6$ was used as the threshold for all binary-like quantitative traits and 91 genes were included in the candidate region significantly associated with ADF. Principal component analysis (PCA) was performedby $R$ package. Genome wide linkage disequilibrium ( $\left.r^{2}\right)$ values of all materials and each subgroup were calculated by TASSEL 3.0 (Bradbury et al., 2007).

\section{Results}

\section{SNPs and Indels identified by re-sequencing}

Genome wide re-sequencing (WGRS) was performed on 206 samples. These included 89 Chinese core collections, 4 local varieties and 113 other collections from different countries and regions, representing the genetic, geographical and morphological diversity of forage sorghum.

After comparing the reference genomesand the SNP calling, we identified 14,570,430 SNPs with MAF $\geq 0.05$ and 1,967,033 indels In principal component analysis, based on all collected SNPs, the first and second principal coordinates (PC) explained $13.16 \%$ and $4.78 \%$ of SNPs molecular variance, respectively (Fig. 1a). Genetic relationship of 206Sorghum reflected the diversity of Sorghum distribution, however the genetic diversity of European varieties is relatively scarce compared with other regions (Fig. 1b).

All materials can be divided into two categories by NJ tree analysis (Fig. 1c). Group囚mainly includes most American accessions and group》 includes sorghum breeding lines from all other regions. Accessions from all breeding lines were mainly but not completely divided into several 


\section{Linkage disequilibrium analysis}

SNP data were used to analyze the genome-wide linkage disequilibrium patterns of Asian and American, Asian $\times$ American, Asian $\times$ Australian and European germplasms, respectively. In the same linkage group, the speed of LD decay can indicate whether the group is selected or not to some extent. Generally speaking, LD of wild population decreased faster than that of domesticated population, and LD of cross pollinated plants decreased faster than that of self pollinated plants. For example, maize: local variety $1 \mathrm{~KB}$, inbred line $2 \mathrm{~KB}$, commercial inbred line $100 \mathrm{~kb}$. In Asia, America, Asia $\times$ America, Asia $\times$ Australia and Europe subgroups, the decay rates are very different, which may lead to different resolution of association mapping in different genome regions. The LD decay rate of European varieties was the lowest, which may be due to self-fertilization(Fig. 1d).

\section{Association analysis of Agronomic Traits}

ADF content of 206 forage sorghum materials showed continuous distribution (Fig. $2 a)$,.A total of 8 candidate regions $(-\log (P)>6)$ spanning $100 \mathrm{~kb}$ were significantly associated with ADF content on chromosomes 2, 5, 6, 7, 8 and 9supported by the strong LD among associated SNPs (Fig. 2band c).

\section{ADF content variation analysis between haplotype}

In order to understand the origin and development of haplotypes, the geographical distribution of SNPS of 8 QTLs in 206 accessions was analyzed (Fig. 3). Allelic distribution for C2_S6746476 show that the 5 accessions contain all haplotypes from AA to TT, ADF content of America×Asia was significantly lower than other accessions, except in genotype of AA,CC,GG,TT. Europe accessions was the opposite (Fig. 3a). For C5_S5473787 genotype of AA, CC, GG, TT, were still high. But among the four genotypes, European sorghum had the lowest ADF content. However, among the other genotypes, European sorghum had the highest ADF content. As a whole, the ADF content of different genotypes was similar (Fig. 3b). For C6_S60364789 the content of ADF in Asia $\times$ Australia sorghum is relatively wide. There were varieties with high and low ADF content. Even some genotypes were higher than Europe sorghum. America and America×Asia sorghum had the lowest ADF content( Fig. 3c). For C7_S60623290 the ADF content of all the haplotypes was similar, In AA, GG, CC and TT haplotypes, the ADF content of European sorghum was still the lowest, but in other haplotypes, the ADF content of AsiaxAustralia sorghum breeding lines were higher than that of European sorghum and became the highest among the five sorghum varieties (Fig. 3d). For C8_S56789460 All haplotypes were classified into two groups: AA, CC, GG, TT and others, The ADF content of these haplotypes were also relatively consistent (Fig. 3e). For C9_S40246128 genotype of $A A, C C, G G$ and $T T, A D F$ content of European sorghum was lower and concentration was relatively concentrated. Among the other genotypes, the ADF content of AsiaxAustralian and European genotypes was the highest, and the difference of ADF content in Australian varieties was large (Fig. 3f).

\section{Genetic collinearity analysis}

The eight candidate regions of ADF content showed about 200000 SNPs on chromosomes 2, 5, 6, 7, 8 and 9 (Table 1), including 91 genes. The results of collinearity analysis showed that 44 of 91 ADF related loci were highly collinearity with previously reported gene families (Table 2). Sorbi_3006G272600 is highly collinear with Osaae3 (Os04g0683700) (Kikuchi s et al. 2003) in rice, which regulates rice blast resistance, floret development and ADF biosynthesis.

Table 1

Candidate QTL regions significantly associated with ADF

\begin{tabular}{|llll|}
\hline Chr & QTL & Start & End \\
\hline 2 & C2_S6746476 & $6,746,476$ & $6,946,476$ \\
\hline 5 & C5_S5473787 & $5,473,787$ & $5,673,787$ \\
\hline 6 & C6_S60364789 & $60,364,789$ & $60,564,789$ \\
\hline 7 & C7_S60623290 & $60,623,290$ & $60,823,291$ \\
\hline 8 & C8_S56789460 & $56,789,460$ & $56,989,479$ \\
\hline 9 & C9_S40246128 & $40,246,128$ & $40,446,128$ \\
\hline
\end{tabular}

Page $4 / 12$ 
Table 2

Gene collinearity of ADF content between sorghum and crops (rice and corn)

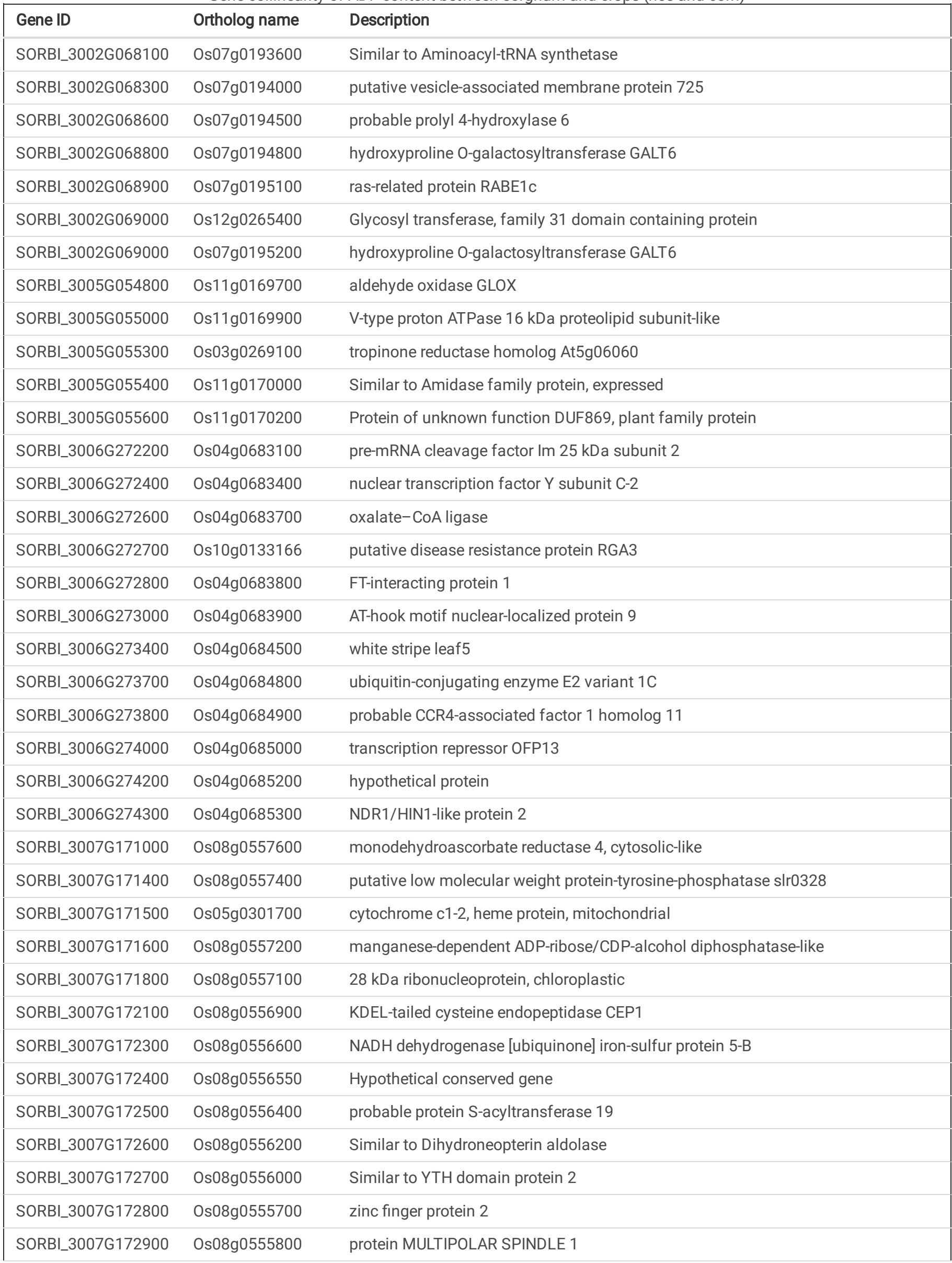




\begin{tabular}{|c|c|c|}
\hline Gene ID & Ortholog name & Description \\
\hline SORBI_3007G173000 & Os08g0555200 & Nonaspanin (TM9SF) family protein \\
\hline SORBI_3007G173000 & Os08g0554900 & transmembrane 9 superfamily member 8 \\
\hline SORBI_3007G173000 & Os08g0555000 & Transmembrane 9 superfamily protein member 2 precursor (p76) \\
\hline SORBI_3002G068100 & Zm00001d018852 & DDT domain-containing protein DDR4 \\
\hline SORBI_3002G068100 & Zm00001d039545 & DDT domain-containing protein DDR4 \\
\hline SORBI_3002G068300 & Zm00001d007767 & vesicle-associated membrane protein 722 \\
\hline SORBI_3002G068300 & Zm00001d019091 & vesicle-associated membrane protein 722 \\
\hline SORBI_3002G068800 & Zm00001d007764 & Hydroxyproline 0-galactosyltransferase GALT4 \\
\hline SORBI_3002G068900 & Zm00001d019093 & Small GTP-binding protein \\
\hline SORBI_3002G069000 & Zm00001d007762 & hydroxyproline 0-galactosyltransferase GALT6 \\
\hline SORBI_3002G069000 & Zm00001d019094 & hydroxyproline 0-galactosyltransferase GALT6 \\
\hline SORBI_3002G069000 & Zm00001d033748 & Hydroxyproline 0-galactosyltransferase GALT4 \\
\hline SORBI_3005G054800 & Zm00001d053008 & glyoxal oxidase \\
\hline SORBI_3005G055000 & Zm00001d004821 & vacuolar ATP synthase $16 \mathrm{kDa}$ proteolipid subunit \\
\hline SORBI_3005G055000 & Zm00001d053010 & V-type proton ATPase $16 \mathrm{kDa}$ proteolipid subunit \\
\hline SORBI_3005G055400 & Zm00001d004817 & Fatty acid amide hydrolase \\
\hline SORBI_3005G055501 & Zm00001d004796 & E3 ubiquitin-protein ligase RZF1 \\
\hline SORBI_3005G055600 & Zm00001d053011 & filament-like plant protein 3 \\
\hline SORBI_3005G056000 & Zm00001d004818 & CBS domain-containing protein CBSCBSPB3 \\
\hline SORBI_3005G056000 & Zm00001d053014 & CBS domain-containing protein CBSCBSPB2 \\
\hline SORBI_3006G272200 & Zm00001d001787 & Pre-mRNA cleavage factor Im $25 \mathrm{kDa}$ subunit 2 \\
\hline SORBI_3006G272400 & Zm00001d026648 & Nuclear transcription factor Y subunit C-4 \\
\hline SORBI_3006G272600 & Zm00001d026649 & Oxalate-CoA ligase \\
\hline SORBI_3006G272800 & Zm00001d001785 & C2 calcium/lipid-binding plant phosphoribosyl transferase family protein \\
\hline SORBI_3006G273000 & Zm00001d001784 & AT-hook motif nuclear-localized protein 5 \\
\hline SORBI_3006G273000 & Zm00001d026652 & AT-hook motif nuclear-localized protein 5 \\
\hline SORBI_3006G273200 & Zm00001d001780 & probable serine/threonine-protein kinase BSK3 \\
\hline SORBI_3006G273200 & Zm00001d026653 & protein kinase APK1B \\
\hline SORBI_3006G273300 & Zm00001d001779 & plant integral membrane protein TIGR01569 containing protein \\
\hline SORBI_3006G273400 & Zm00001d026654 & Pentatricopeptide repeat-containing protein chloroplastic \\
\hline SORBI_3006G273800 & Zm00001d001774 & hypothetical protein \\
\hline SORBI_3006G273900 & Zm00001d001773 & pentatricopeptide repeat-containing protein At3g51320 \\
\hline SORBI_3006G274000 & Zm00001d026661 & Transcription repressor OFP13 \\
\hline SORBI_3006G274000 & Zm00001d026663 & hypothetical protein \\
\hline SORBI_3006G274200 & Zm00001d001771 & aspartic proteinase nepenthesin-2 \\
\hline SORBI_3006G274300 & Zm00001d001772 & harpin inducing protein \\
\hline SORBI_3006G274300 & Zm00001d026662 & hypothetical protein \\
\hline SORBI_3007G170900 & Zm00001d005344 & histidine-containing phosphotransfer protein 2 \\
\hline
\end{tabular}




\begin{tabular}{|lll|}
\hline Gene ID & Ortholog name & Description \\
\hline SORBI_3007G170900 & Zm00001d049952 & his-containing phosphotransfer protein \\
\hline SORBI_3007G171000 & Zm00001d049954 & hypothetical protein \\
\hline SORBI_3007G171100 & Zm00001d049958 & Putative peptidyl-prolyl cis-trans isomerase and WD40 repeat domain family protein \\
\hline SORBI_3007G171400 & Zm00001d031963 & Low molecular weight protein-tyrosine-phosphatase slr0328 \\
\hline SORBI_3007G171400 & Zm00001d046049 & protein tyrosine phosphatases;protein tyrosine phosphatases \\
\hline SORBI_3007G171500 & Zm00001d031967 & cytochrome c1, heme protein \\
\hline SORBI_3007G171600 & Zm00001d049960 & manganese-dependent ADP-ribose/CDP-alcohol diphosphatase \\
\hline SORBI_3007G171800 & Zm00001d031969 & RNA-binding protein CP31B chloroplastic \\
\hline SORBI_3007G172100 & Zm00001d031971 & Vignain \\
\hline SORBI_3007G172300 & Zm00001d031975 & Fb14 \\
\hline SORBI_3007G172400 & Zm00001d049974 & peroxisome biogenesis protein 1 \\
\hline SORBI_3007G172500 & Zm00001d031977 & putative protein S-acyltransferase 19 \\
\hline SORBI_3007G172600 & Zm00001d031979 & dihydroneopterin aldolase 1 \\
\hline SORBI_3007G172600 & Zm00001d031995 & Dihydroneopterin aldolase 2 \\
\hline SORBI_3007G172700 & Zm00001d031981 & evolutionarily conserved C-terminal region 5 \\
\hline SORBI_3007G172800 & Zm00001d031985 & protein LATE FLOWERING \\
\hline SORBI_3007G172900 & Zm00001d050498 & multipolar spindle 1 \\
\hline SORBI_3007G173000 & Zm00001d031986 & transmembrane 9 superfamily member 9 \\
\hline
\end{tabular}

\section{Discussion}

The improvement of crop productivity is based on the aggregation of good traits of different materials into one variety. However, compared with rice (Huang et al. 2010) and maize (Jiao et al. 2012), the availability of sorghum genome resources for genetic analysis of quality traits is little relatively. The research on the huge genetic diversity of Sorghum bicolor is following up (Zheng et al. 2011; Morris et al. 2013; Ordonia et al. 2016). Systematic evaluation and rational utilization of germplasm resources play a key role in the improvement of Forage Sorghum Germplasm resources. Through the rational utilization of breeding materials, ideal varieties with allelic variation can be obtained, such as biomass, protein content and ADF content.In this study, we generated 14,570,430 SNPs and 1,967,033 indels, which created a valuable resource for Sorghum bicolor, phenotype research, molecular breeding and related species in the future.

The whole genome SNPs were used to analyze the population structure and the genetic relationship between the 206 Sorghum accessions. The genetic relationship among 206 sorghum materials showed that the distribution of sorghum materials, breeding lines and their improved varieties in Asia and America were more diversified, while the genetic diversity of European materials wrer relatively limited. These results increase our understanding of crop evolution within and across agroclimatic regions in America, Asia and Europe.

Sorghum genome data is gradually enriched in the continuous research of scholars in various countries, which would increase our understanding of allelic variation in the collection of genetic resources, and also encourage breeders in various countries to cultivate more excellent varieties. We identified 8 QTLs related to ADF content and observed the characteristics of multiple alleles of ADF content in sorghum varieties from different regions (Fig. $3 a-f$ ). All the genotypes can be divided into two groups. The ADF content of varieties with AA, CC, GG and TT haplotypes were significantly higher than that of other haplotypes. This indicated that homozygous haplotypes did not contain favorable genes for reducing ADF content due to more selfing and less hybridization. However, among the homozygous haplotypes, the content of ADF in European sorghum was the lowest (fig. a-f). On the other hand, the ADF contents of American, Asian, American $\times$ Asian and American $\times$ Australian sorghum were lower than that of European sorghum, which was opposite to that of homozygous sorghum. However, no matter homozygous allele or heterozygous allele of European sorghum, the content of ADF did not change much, which was due to the small number of samples and simple genetic evolution background. We revealed that 44 of 91 genes related to ADF content in sorghum were highly collinear with those previously reported in rice and maize(Table 2). SORBI_3006G272600 is highly collinear with OsAAE3 (Os04g0683700) (Kikuchi S et al.2003) in rice, which regulates rice blast resistance, floret development, and ADF biosynthesis. This information will guide breeders to develop more accurate breeding strategies and further improve the quality of sorghum varieties. 
Forage sorghum has the characteristics of high biomass, good stress resistance, juicy stem, high nutrient utilization efficiency, and is one of the main biomass raw materials for animal husbandry. The results showed that ADF affected the absorption of nutrients in feed by cattle, sheep,

pigs and other livestock. The reasonable proportion of ADF in feed could improve the nutrient absorption rate of feed animals, and thus improve meat quality(Zhang et al,2020; Zheng et al. 2007 ; Luo et al. 2000; Yuan et al. 1999; Yu et al.2018). In forage sorghum breeding, we hope to select varieties with moderate acid detergent fiber is desired. The MTAs and materials with ideal alleles found in this study will help to accelerate the improvement of forage sorghum varieties and hybrids and make the ADF content reach the ideal range. In order to better understand the potential regulatory mechanism of ADF content, further studies are needed to identify these and other MTAs related genes, which in turn will contribute to the breeding of excellent forage sorghum varieties.

In conclusion, the genomic data of Forage Sorghum collected all over the world, the inferences from the analysis and the MTAs related to agronomic traits provide valuable resources for promoting forage sorghum variety improvement. The study of candidate genes related to ADF content lays a foundation for the potential application of functional genomics methods in verifying the function and role of candidate genes in determining ADF content.

\section{Declarations}

\section{Funding}

This work was financially supported by Agriculture Research System of China (Grant No. CARS-06)

\section{Ethics approval and consent to participate}

Not applicable.

\section{Compliance with ethical standards}

The authors declare that all experiments from this study comply with laws and regulations of PR China.

\section{Competing interests}

The authors declare that they have no competing interests.

Authors' contributions $\mathrm{HN}$ is mainly responsible for the implementation of experimental design, data analysis and paper writing. $\mathrm{YH}$ helped to design the experimental scheme, JP provided financial support, and YW, XL and JC cooperated with HN in field investigation and sampling. All authors have read the manuscript and agree with all the conclusions and opinions

\section{References}

1. Boddu J, Svabek C, Sekhon R, Gevens A, Nicholson RL, Jones AD, Pedersen JF, Gustine DL, Chopra S (2004) Expression of a putative flavonoid 3'-hydroxylase in sorghum mesocotyls synthesizing 3-deoxyanthocyanidin phytoalexins. Physiological and Molecular Plant Pathology 65(2), 101-113

2. Bouchet S, Pot D, Deu M, Rami JF, Billot C, Perrier X, Rivallan R, Gardes L,Xia L,Wenzl P, Kilian A, Glaszmann JC (2012) Genetic structure, linkage disequilibrium and signature of selection in Sorghum: lessons from physically anchored DarT markers. PLoS One 7:e33470

3. Bouchet S, Olatoye MO, Marla SR, Perumal R, Tesso T, Yu J, Tuinstra M, Morris GP (2017) Increased power to dissect adaptive traits in global sorghum diversity using a nested association mapping population. Genetics 206(2),573-585.

4. Bradbury PJ, Zhang Z, Kroon DE, Casstevens TM, Ramdoss Y, Buckler ES (2007) TASSEL: software for association mapping of complex traits in diverse samples. Bioinformatics 23,2633-2635

5. Casa AM, Mitchell SE, Jensen J, Hamblin MT, Paterson AH, Aquadro CF, Kresovich S (2006) Evidence for a selective sweep on chromosome 1 of cultivated sorghum. Crop Science 46, S27-S40

6. Casa AM, Pressoir G, Brown PJ, Mitchell SE, Rooney WL, Tuinstra MR, Franks CD, Kresovich S (2008) Community resources and strategies for association mapping in sorghum. Crop Science 48,30-40

7. Endelman JB, Jannink JL (2012) Shrinkage estimation of the realized relationship matrix. G3-Genes Genomes Genetics 2(11),1405-1413

8. Font R, Wittkop B, Badani A, Celestino M, Friedt W, Lühs W; De Haro-Bailón A(2015) The measurements of acid detergent fibre in rapeseed by visible and near-infrared spectroscopy Plant Breeding 124(4),410-412

9. Han F, Ullrich S, Romagosa I, Clancy JA, Froseth JA, Wesenberg DM (2003) Quantitative genetic analysis of acid detergent fibre content in barley grain Journal of Cereal Science, 38(2),167-172.

10. He Z (2011) International standard for determination of acid detergent fiber and lignin Sci tech outlook. (6),31-32

Page $8 / 12$ 
11. Huang X, Wei X, Sang T, Zhao Q, Feng Q, Zhao Y, Li C, Zhu C, Lu T, Zhang Z, Li M, Fan D, Guo Y, Wang A, Wang L, Deng L, Li W, Lu Y, Weng Q, Liu K, Huang T, Zhou T, Jing Y, Li W, Lin Z, Buckler ES, Qian Q, Zhang QF, Li J, Han B (2010) Genome-wide association studies of 14 agronomic traits in rice landraces. Nature Genetics 42,961-967

12. Jiao Y, Zhao H, Ren L, SongW, Zeng B, Guo J, Wang B, Liu Z, Chen J, Li W, Zhang M, Xie S, Lai J (2012) Genome-wide genetic changes during modern breeding ofmaize. Nature Genetics 44,812-815

13. Jiao Y, Peluso P, Shi J, Liang T, Stitzer M, Wang B, Campbell M, Stein J, Wei X, Chin C, Guill K, Regulski M, Kumari S, Olson A, Gent J, Schneider K, Wolfgruber T, May M, Springer M, Antoniou E, McCombie W, Presting G, McMullen M(2017) Improved maize reference genome with single-molecule technologies Naure Jun 22;546(7659),524-527.

14. Kikuchi S, Satoh K, Nagata T, Kawagashira N, Doi K, Kishimoto N, Yazaki J, Ishikawa M, Yamada H, Ooka H, Hotta I, Kojima K, Namiki T, Ohneda E, Yahagi W, Suzuki K, Chao J, Ohtsuki K, Shishiki T (2003) Collection, mapping, and annotation of over 28,000 cDNA clones from japonica rice. Science 301: 376-379.

15. Li J, Sun L, Li Y, Lu Y, Pan X, Zhang X, Liu Y, Song Z (2018) Rapid prediction of acid detergent fiber content in corn stover based on NIRspectroscopy technology. Optik - International Journal for Light and Electron Optics 180(1),34-45

16. Liu Q, Luo L, Zheng L (2018) Lignins: biosynthesis and biological functions in plants. Int J Molecular Science 19:335

17. Ma P, Zhang X, Luo B, Chen Z, He X, Zhang H, Li B, Liu D, Wu L, Gao S, Gao D, Zhang S, Gao S (2021) Transcriptomic and genome-wide association study reveal long noncoding RNAs responding to nitrogen deficiency in maize. BMC Plant Biology 21(1)

18. Mathur S, Umakanth AV, Tonapi VA, Sharma R, Sharma MK (2017) Sweet sorghum as biofuel feedstock: recent advances and available resources. Biotechnol Biofuels 10:146

19. Miranda A, Tirado-González DN, Tirado-Estrada G, Améndola-Massiotti RD, Sandoval-González L, Ramírez-Valverde R, Salem A(2020) Quantifying non-fibrous carbohydrates, acid detergent fiber and cellulose of forage through an in vitro gas production technique. Journal of the Science of Food and Agriculture 2(2).

20. Morris GP, Ramu P, Deshpande SP, Hash CT, Shah T, Upadhyaya HD, Riera-Lizarazu O, Brown PJ, Acharya CB, Mitchell SE, Harriman J, Glaubitz JC, Buckler ES, Kresovich S (2013) Population genomic and genome-wide association studies of agroclimatic traits in sorghum. Proceedings of the National Academy of Sciences of the United States of America, 2013. 110,453-458

21. Mundia CW, Secchi S, Akamani K, Wang G (2019) A regional comparison of factors affecting global sorghum production: the case of North America, Asia and Africa's Sahel. Sustainability 11:2135

22. National Research Council (1996) Lost crops of Africa, vol 1. Natl Acad Press, Washington, DC

23. Nida H, Girma G, Mekonen M, Tirfessa A, Seyoum A, Bejiga T, Birhanu C, Dessalegn K, Senbetay T, Ayana G, Tesso T, Ejeta G, Mengiste T (2021). Genome-wide association analysis reveals seed protein loci as determinants of variations in grain mold resistance in sorghum Theoretical and Applied Genetics 134 1-18

24. Ordonia R, Ito Y, Morinaka Y, Sazuka T, Matsuaka M (2016) Molecular breeding of Sorghum bicolor, a novel energy crop. International Review of Cell and Molecular Biology 321,221-257

25. Ping J, Zhang F, Niu H, Yang H, Lv X, Du Z, Li H, Wang Y (2018) Genetic diversity analysis of germplasm resources of forage Sorghum based on SSR marker. Molecular Plant Breeding 14: 4663-4670

26. Ross-lbarra J, Dawe R, Hastie A, Rank D, Ware D Improved maize reference genome with single molecule technologies. Nature, 2017, 546(7659),524-527.

27. Sara O, RafaelM, María J, Elena C, Antonio D (2019) Analysis of the Acid Detergent Fibre Content in Turnip Greens and Turnip Tops (Brassica rapa L. Subsp. rapa) by Means of Near-InfraredReflectance. Foods 8(9). 364.

28. Shoemaker C, Bransby D (2010) The role of sorghum as a bioenergy feedstock. Sustainable alternative fuel feedstock opportunities, challenges and roadmaps for six US regions. Soil and Water Conservation Society, Atlanta, 149-159

29. Van Soest PJ, Robertson JB, Lewis BA (1991). Methods for dietary fiber, neutral detergent fiber, and nonstarch polysaccharides in relation to animal nutrition. Journal of Dairy Science, 74(10), 3583-3597.

30. Verma Rahul K.; Chetia S.K.; Dey P.C.; Rahman Anjum; Saikia Sandhani; Sharma Vinay; Sharma Himanshu; Sen P.; Modi M.K(2021) Genome-wide association studies for agronomical traits in winter rice accessions of Assam. Genomics .113(3),1037-1047

31. Vermerris W (2011) Survey of genomics approaches to improve bioenergy traits in maize, sorghum and sugarcanefree access. journal of Integrative Plant Biology, 53(2),105-119.

32. Yu Z, Song C, He B, Yang W, Jiang J (2017) Effects of ADF level on nutrient digestibility and nitrogen balance between two breeds of pig. Chinese journal of animal science 053(012):56-61.

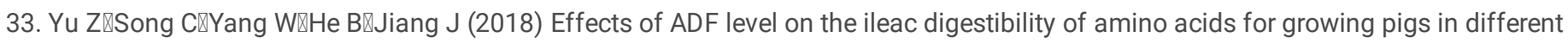
breeds. China Feed 597(01):32-36. 
34. Zhang S, Jing Z, Wu F, Luan R, Wang J, Zhang Y, Wang X (2018) The agronomic characters and SRAP genetic diversity anal- ysis of 21 introduced male sterile lines of brown midrib forage sorghum. Pratacultural Science 35:1072-1079

35. Zhang Z, Li P, Liu L, Zhang S, Li J, Zhang L, Li D (2020) Ether extract and acid detergent fibre but not glucosinolates are determinants of the digestible and metabolizable energy of rapeseed meal in growing pigs. Journal of Applied Animal Research 48(1),384-389.

36. Zheng L, Guo X, He B, Sun L, Peng Y, Dong S, Liu T, Jiang S, Ramachandran S, Liu C, Jing H (2011) Genome-wide patterns of genetic variation in sweet and grain sorghum (Sorghum bicolor). Genome Biology 12:R114

\section{Figures}

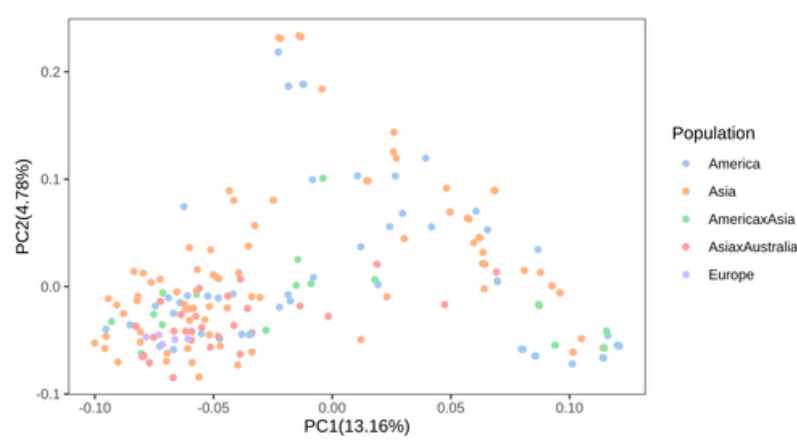

A)
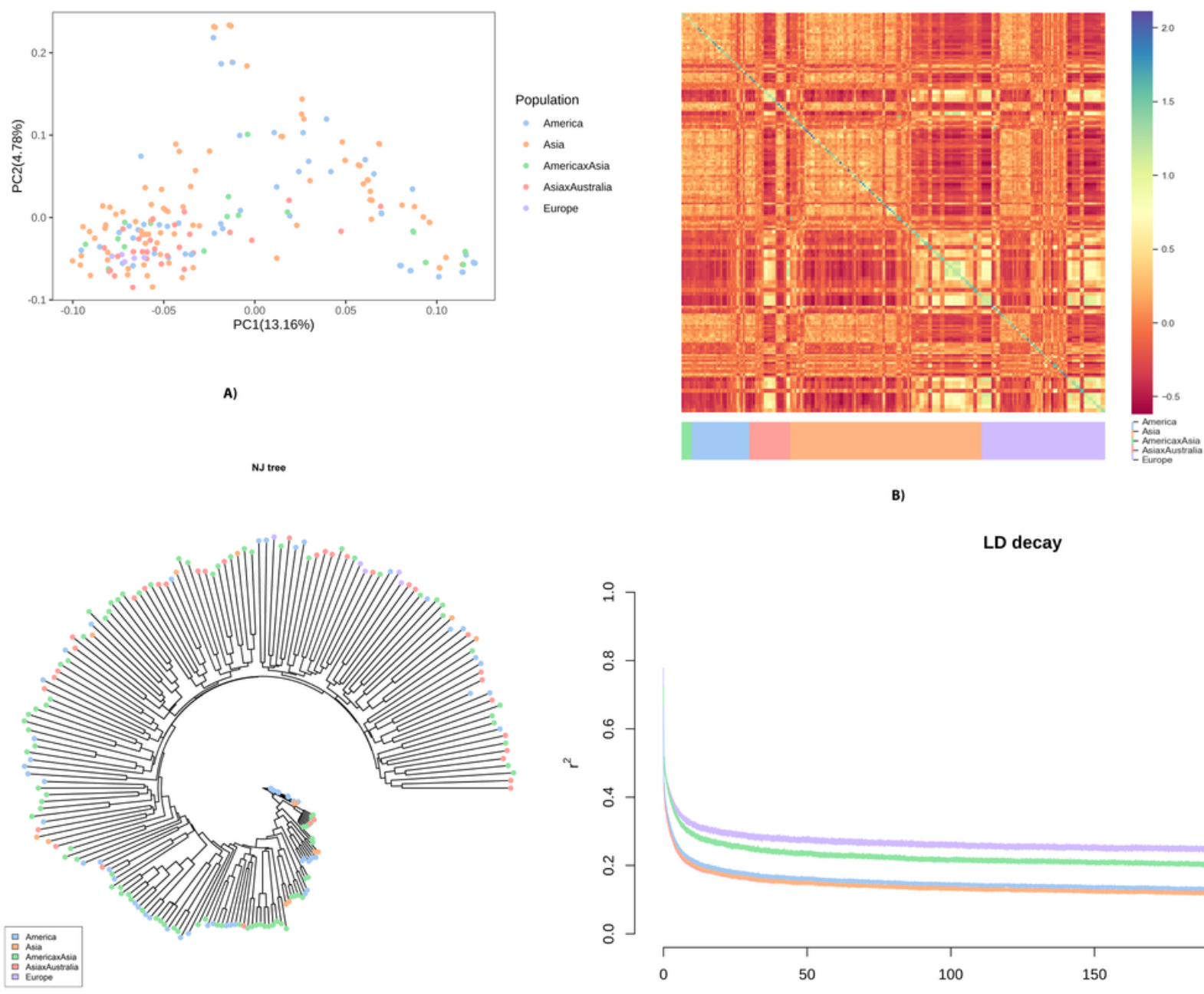

C)

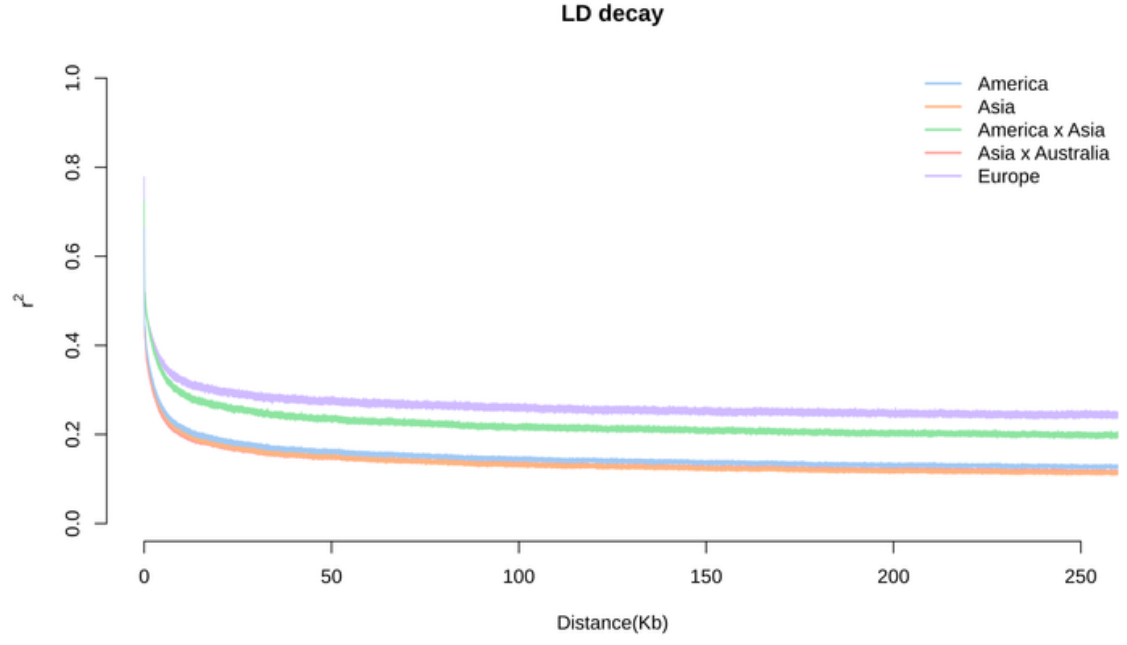

D)

\section{Figure 1}

Genetic structure of 206 sorghum accessions using SNPs detected in whole-genome resequencing data. a PCA, b genetic relatedness using SNPs detected in whole-genome resequencing data, c NJ tree constructed from simple matching distance of all SNPs, and d LD decay 


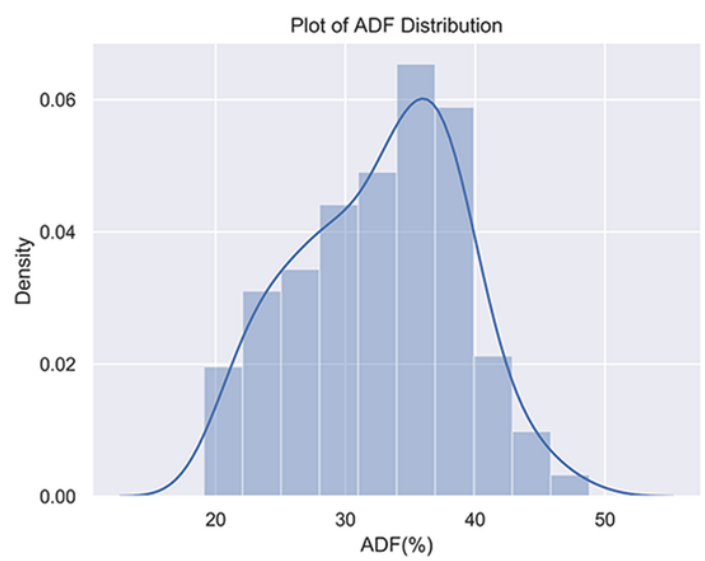

A)

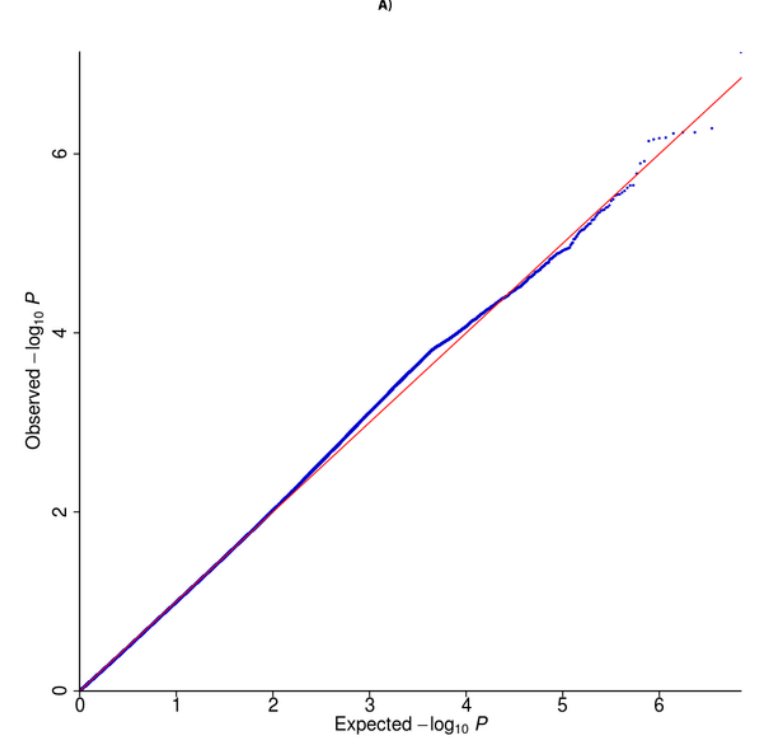

c)

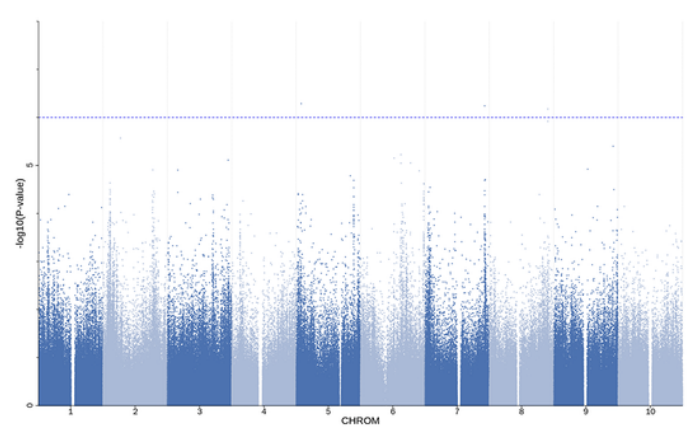

B)

Figure 2

GWAS of the sorghum ADF content. a Distribution of the ADF content values. b Manhattan plot for ADF content. The -log10 P values from a genome-wide scan are plotted against the position on each of the 10 chromosomes. c Quantile plot for ADF content. 

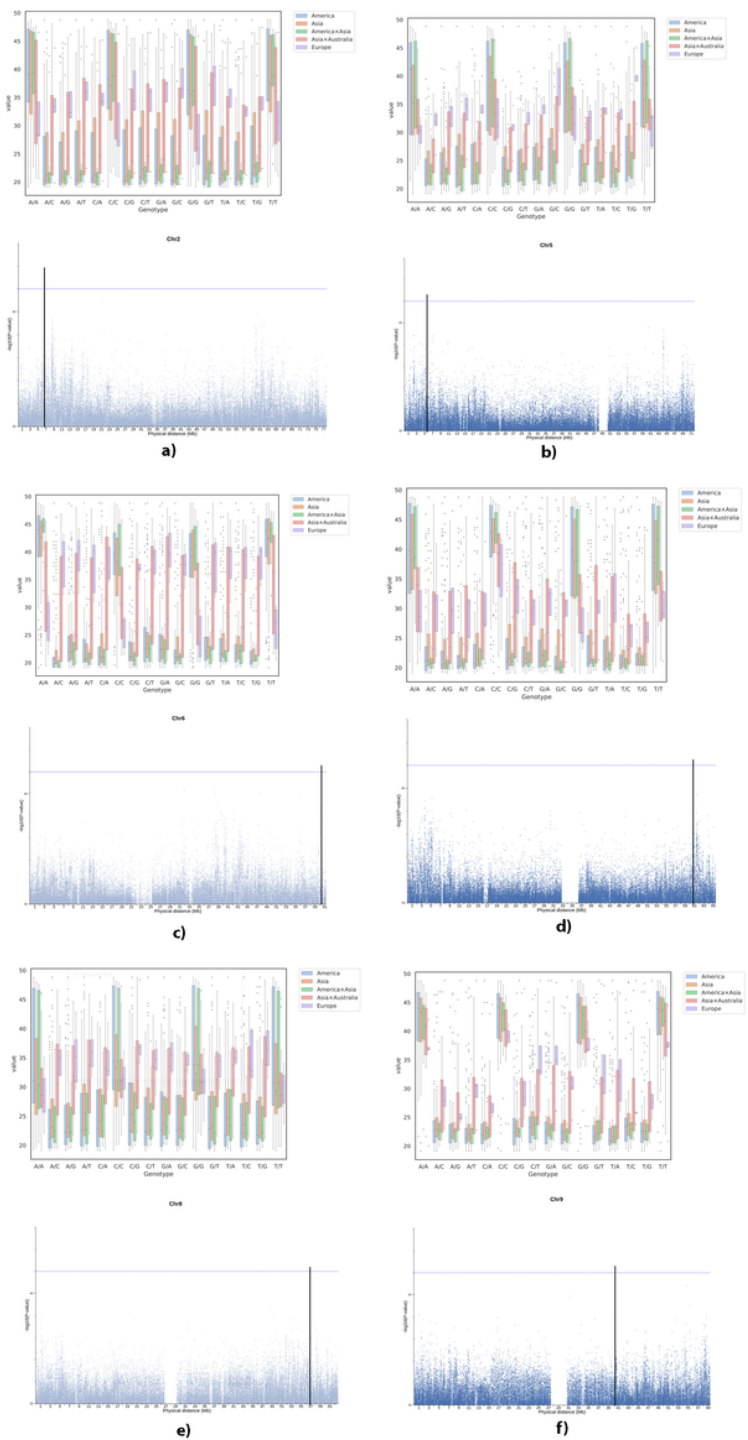

Figure 3

The geographic distribution of alleles and ADF content variation among different haplotypes in each QTL. a C2_S6746476. b C5_S5473787. c C6_S60364789.d C7_S60623290.e C8_S56789460.f C9_S40246128. 